\title{
Single maintenance and reliever therapy (SMART) of asthma: a critical appraisal
}

\author{
Kenneth R Chapman, ${ }^{1}$ Neil C Barnes, ${ }^{2}$ Andrew P Greening, ${ }^{3}$ Paul W Jones, ${ }^{4}$ \\ $S$ Pedersen ${ }^{5}$
}

${ }^{1}$ Asthma and Airway Centre University Health Network, Toronto Western Hospital, Toronto, Ontario, Canada ${ }^{2}$ Department of Respiratory Medicine, London Chest Hospital, London, UK ${ }^{3}$ Respiratory Unit, Western General Hospital, Edinburgh, UK ${ }^{4}$ Department of Respiratory Medicine, St George's, University of London, London, UK

${ }^{5}$ Pediatric Research Unit, Kolding Hospital, Kolding, Denmark

\section{Correspondence to}

Kenneth R Chapman, Asthma and Airway Centre, University Health Network, Toronto Western Hospital, Room 7-451 East Wing, 399 Bathurst St, Toronto, Ontario M5T 2S8, Canada;

kchapman@ca.inter.net

Received 30 October 2009 Accepted 28 February 2010

\section{(2) UNLOCK:}

This paper is freely available online under the BMJ Journals unlocked scheme, see http:// thorax.bmj.com/site/about/ unlocked.xhtml

\section{ABSTRACT}

The use of a combination inhaler containing budesonide and formoterol as both maintenance and quick relief therapy (SMART) has been recommended as an improved method of using inhaled corticosteroid/longacting $\beta$ agonist (ICS/LABA) therapy. Published doubleblind trials show that budesonide/formoterol therapy delivered in SMART fashion achieves better asthma outcomes than budesonide monotherapy or lower doses of budesonide/formoterol therapy delivered in constant dosage. Attempts to compare budesonide/formoterol SMART therapy with regular combination ICS/LABA dosing using other compounds have been confounded by a lack of blinding and unspecified dose adjustment strategies. The asthma control outcomes in SMARTtreated patients are poor; it has been reported that only $17.1 \%$ of SMART-treated patients are controlled. In seven trials of 6-12 months duration, patients using SMART have used quick reliever daily (weighted average 0.92 inhalations/day), have awakened with asthma symptoms once every 7-10 days (weighted average $11.5 \%$ of nights), have suffered asthma symptoms more than half of days (weighted average $54.0 \%$ of days) and have had a severe exacerbation rate of one in five patients per year (weighted average 0.22 severe exacerbations/patient/year). These poor outcomes may reflect the recruitment of a skewed patient population. Although improvement from baseline has been attributed to these patients receiving additional ICS therapy at pivotal times, electronic monitoring has not been used to test this hypothesis nor the equally plausible hypothesis that patients who are non-compliant with maintenance medication have used budesonide/formoterol as needed for self-treatment of exacerbations. Although the longterm consequences of SMART therapy have not been studied, its use over 1 year has been associated with significant increases in sputum and biopsy eosinophilia. At present, there is no evidence that better asthma treatment outcomes can be obtained by moment-tomoment symptom-driven use of ICS/LABA therapy than conventional physician-monitored and adjusted ICS/LABA therapy.

\section{INTRODUCTION}

For patients whose asthma remains uncontrolled despite the regular inhalation of corticosteroid alone, the inhalation of corticosteroid coupled with long-acting $\beta_{2}$ agonist (ICS/LABA) from a single inhalation device twice daily has become a valuable strategy. ${ }^{1}$ This combination approach reduces exacerbation risk and increases the likelihood of controlling asthma more often, more rapidly and at a lower dose of ICS than is seen with ICS therapy alone. $^{2-4}$ Typically, combination ICS/LABA therapy has been prescribed with a separate rapidacting $\beta$ agonist inhaler used for relief of occasional breakthrough symptoms, but combination ICS/ LABA formulations employing formoterol as the LABA component allow patients to employ their usual maintenance inhaler for quick relief as well. This strategy of medication use has been recognised by regulatory authorities and in international guidelines. ${ }^{1}$ It has recently been argued that a strategy of using single maintenance and reliever therapy (SMART) offers more than convenience to patients; it is said to provide better improvements in several outcomes with lower ICS dosing than the traditional combination therapy approach of constant maintenance dosing with a separate reliever. $^{5-7}$ Since these recommendations were made, the SMART strategy studies have been reviewed critically and independently as part of the Cochrane process, the impact of SMART therapy upon airways inflammation has been reported and the limitations of the research evidence have been highlighted. 89 Given these emerging concerns, the following review examines critically the studies that have tested the SMART strategy to determine the strength of evidence supporting its potential benefits, its applicability to the general asthma population and the questions that remain unanswered.

\section{CLINICAL STUDIES \\ Double-blind studies}

Three double-blind studies explored the SMART strategy in ICS-treated but poorly controlled patients. ${ }^{10-12}$ These studies compared SMART with conventional ICS monotherapy with budesonide alone or, in one study, with conventional combination therapy with budesonide/formoterol and a separate short-acting $\beta$ agonist (SABA) reliever (figure 1).

Rabe and colleagues assigned patients to 6 months of either budesonide/formoterol combination inhaler (Symbicort 100/6) one puff twice daily and as needed up to 10 puffs per day or budesonide $200 \mu \mathrm{g}$ (Pulmicort) one puff twice daily with terbutaline $500 \mu \mathrm{g} /$ puff (Bricanyl) as needed. ${ }^{10}$ In contrast to almost all subsequent SMART studies, the primary outcome variable was a measure of lung function rather than time to first severe exacerbation. Morning peak expiratory flow (PEF) improved significantly more in patients treated with ICS/LABA than in those treated with ICS alone. Other outcome variables similarly favoured the ICS/LABA group including evening 

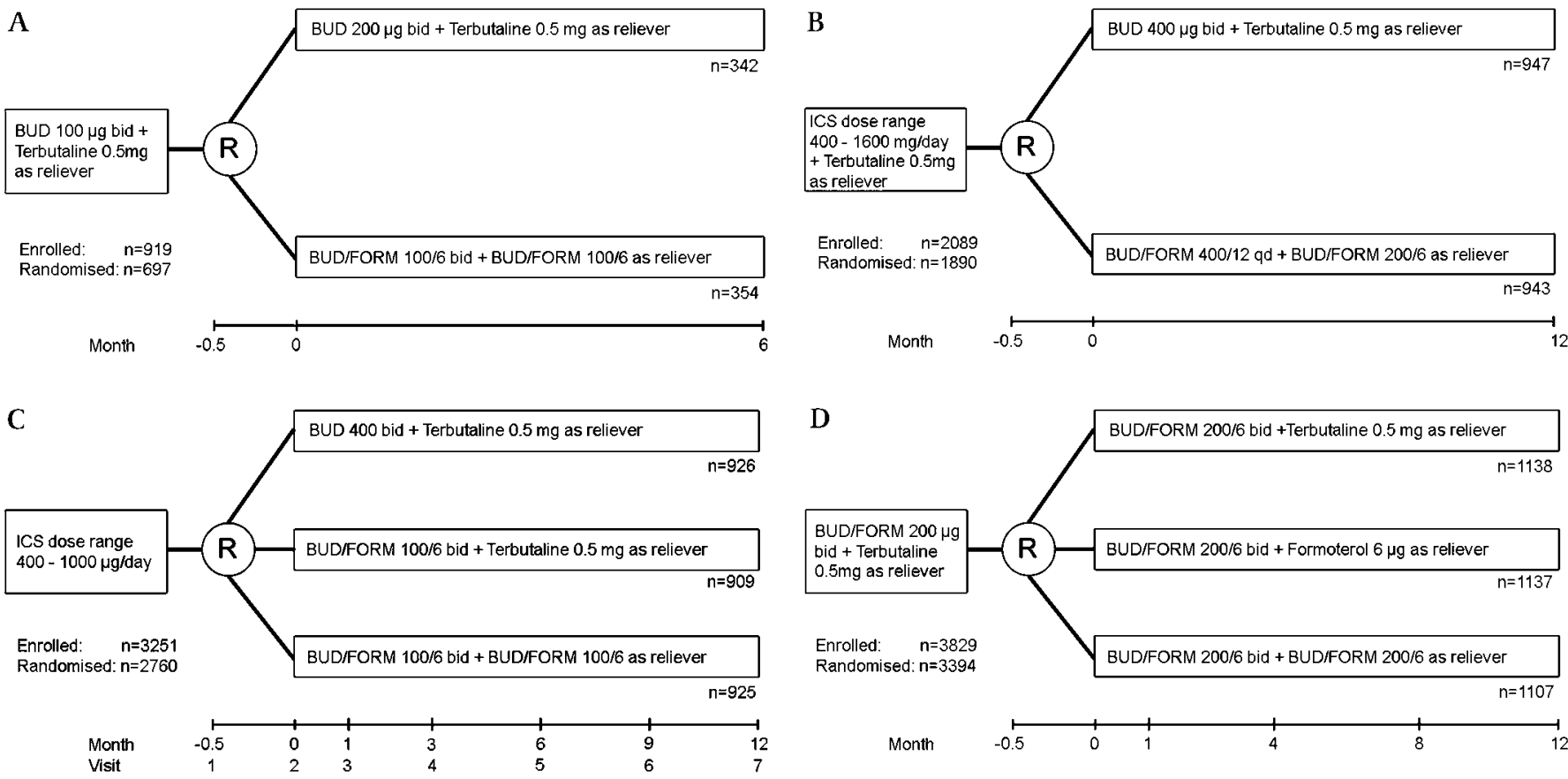

Figure 1 Study designs for double-blind single maintenance and reliever therapy (SMART) studies. (A) Comparison of inhaled corticosteroid (ICS) monotherapy with ICS/long-acting $\beta$ agonist (LABA). ${ }^{15}$ (B) Comparison of ICS monotherapy with ICS/LABA. ${ }^{16}$ (C) Comparison of ICS monotherapy with two doses of ICS/LABA, SMART being the higher dosage ICS/LABA treatment arm. ${ }^{17}$ (D) Comparison of three ICS/LABA treatment arms: with shortacting $\beta_{2}$ agonist, with additional LABA or with additional ICS and additional LABA. ${ }^{19}$ BUD, budesonide; FORM, formoterol.

PEF, reliever-free days, as-needed reliever use, asthma symptom score, symptom-free days and asthma control days. Also noted was a reduced 'severe' exacerbation rate in patients treated with ICS/LABA. The definition of severe exacerbation was expanded in this study beyond the usual clinical meaning to include not only a hospitalisation or emergency department visit but also a short course of prednisone or a decrease in morning PEF $\geq 30 \%$ from baseline on two or more consecutive days. Indeed, 57 of 66 exacerbations defined by the fall in morning PEF had not been detected clinically by the patient or investigator. Defining an exacerbation based solely on PEF changes has been questioned in a recent review and the ATS/ERS statement concerning the definition of exacerbations for asthma research. ${ }^{13}$ The pattern of reliever use was examined in the SMART group; despite $6 \%$ of patients using $>8$ puffs of ICS/LABA as reliever at least once during the trial and $3 \%$ using $>10$ puffs on at least one day, no adverse events were attributed to such usage although three episodes of tachycardia were reported in the SMART group and none in the budesonide monotherapy group. Other safety parameters were not significantly different between groups.

Scicchitano and colleagues undertook a study of similar design to the study by Rabe et al but the study was of 12 months duration and time to first severe exacerbation (using the expanded definition) was the primary outcome variable. ${ }^{11}$ One arm was treated with budesonide/formoterol 200/6 (Symbicort 200/6) once daily with additional doses as needed, the other received budesonide $200 \mu \mathrm{g}$ (Pulmicort 200) two puffs twice daily with terbutaline $500 \mu \mathrm{g} /$ puff reliever as needed. The results were similar to those reported by Rabe and colleagues. Time to first severe exacerbation was longer in the ICS/LABA combination therapy group while other symptom outcomes were also better. Most adverse events and safety events were similar between treatment groups, although one patient in the SMART group developed atrial fibrillation attributed to study medication by the investigator.
The results of these two early SMART trials do not elucidate a unique value of a SMART approach; they confirm only that the addition of a long-acting $\beta_{2}$ agonist to inhaled steroids is an effective option.

The largest of the early trials was reported by O'Byrne and colleagues. ${ }^{12}$ This 1 -year trial assigned patients to (1) budesonide/formoterol $100 / 6$ one puff twice daily with terbutaline reliever; (2) budesonide $400 \mu \mathrm{g}$ twice daily with terbutaline reliever; or (3) budesonide/formoterol 100/6 one puff twice daily with additional doses as reliever. Time to first severe exacerbation was delayed in the SMART group compared with the other regimens. Secondary outcome variables were also better. The investigators suggested the timing of additional ICS therapy in the SMART group as a possible mechanism to explain the better outcomes with SMART versus budesonide monotherapy at mean daily doses of $300 \mu \mathrm{g}$ versus $400 \mu \mathrm{g}$ respectively. They did not discuss the improved outcome of SMART versus conventional combination therapy with SABA reliever, but their data showed that patients on SMART averaged $50 \%$ higher daily ICS doses than patients using conventional combination therapy. Thus, this trial reconfirms that increasing the dose of ICS may be helpful in improving asthma outcomes.

A later blinded trial by Rabe and colleagues compared two conventional combination treatment arms with a SMART treatment arm over 12 months (figure 1). ${ }^{14}$ Patients with poorly controlled asthma were randomised to budesonide/formoterol 200/6 (Symbicort 200) one puff twice daily and one of terbutaline $500 \mu \mathrm{g}$, formoterol $6 \mu \mathrm{g}$ or budesonide/formoterol 200/6 as reliever. The time to first severe exacerbation (defined as hospitalisation, emergency department visit or prednisone use) was longest in the SMART arm, shorter in the formoterol reliever arm and shortest in the terbutaline reliever arm. Although several individual symptom outcomes were statistically better in the SMART arm than in the comparator arms, there was no difference among treatments in the asthma control days or the 
quality of life. The average daily dose of budesonide was increased by $50 \%$ in the SMART-treated patients compared with the comparator arms (604 $\mu \mathrm{g}$ vs $400 \mu \mathrm{g})$.

In aggregate, these blinded studies showed no clear safety signals for SMART therapy. They confirmed that combination therapy with ICS/LABA produces better asthma outcomes than ICS monotherapy and that higher doses of ICS/LABA combination therapy produces better results than lower doses.

\section{Comparative studies}

Several studies have compared SMART with budesonide/ formoterol with conventional combination therapy with salmeterol/fluticasone. Such studies have been confounded by a lack of blinding and several elements of trial design that subtly disadvantaged the non-SMART arm. For example, Vogelmeier and colleagues reported fewer severe exacerbations in patients treated with budesonide/formoterol in SMART fashion compared with patients treated with fluticasone/salmeterol and separate salbutamol reliever. ${ }^{15}$ However, the authors acknowledged that 'as titration of maintenance medication was left to physician judgement and was not protocol driven, patients could have been inadvertently undertreated'. A majority of patients $(62 \%)$ were using budesonide (most likely in Turbuhaler format) at the time of recruitment; it would be difficult to exclude bias in the study with patients assigned to their familiar inhaler or a novel inhaler in open-label fashion. The use of devices that require different inhalation techniques (dry powder Turbuhaler SMART arm versus metered dose aerosol comparator arm) further complicates interpretation. Moreover, the investigators chose to report the doses of inhaled medication differently between arms, using lower ex-actuator values for the SMART arm and higher ex-valve values for the comparator arm. Although this and several other studies have suggested lower ICS exposure for patients using a SMART regimen, this conclusion has been based on patient diary reports of medication use and has not been validated by demonstrating differences in urinary cortisol values or other pharmacodynamic end points.

Sears et al have also reported a study in which the management of the comparator arm was undefined. ${ }^{16}$ Patients with poorly controlled asthma were recruited from community practices. Some participating practices were asked to instruct patients in the correct use of a Turbuhaler and to switch these patients to SMART therapy while other practices were left to manage patients using 'current best practice', a strategy that was unspecified. Despite failing to show significant differences between treatments in the primary outcome variable (time to first severe exacerbation) and almost all secondary outcome variables (including hospitalisation rates, emergency department visits, PEF, Asthma Control Questionnaire (ACQ)-5 and sputum eosinophil counts), the investigators calculated the lower medication costs in the SMART group and reported the statistical significance of the finding. Similar negative findings (but without sputum eosinophil assessment) are available online from two unpublished European studies. ${ }^{17} 18$

In a blinded study, Bousquet and colleagues also failed to find a significant difference between budesonide/formoterol SMART therapy and constant dose fluticasone/salmeterol therapy in their primary outcome variable, time to first severe exacerbation and multiple secondary outcomes including reliever use, percentage of reliever-free days, asthma symptom scores, percentage of symptom-free days, nocturnal awakenings, asthma control days, ACQ-5 and morning and evening PEF. ${ }^{19}$ Time to first plus second exacerbations was significantly lower in the budesonide/formoterol group than in the fluticasone/ salmeterol group. This study differed from others in attempting to double-blind the study using double dummy techniques, although it seems probable that patients would have been able to distinguish bronchodilator-containing inhalers from placebo inhalers. Terbutaline was the reliever medication in the nonSMART arm rather than formoterol, an important difference given the different efficacy and side effect profiles between these bronchodilators. Two other studies have compared the as-needed use of these two bronchodilators in a population of patients with asthma characteristics similar to those in the study by Bousquet et al and reported a significantly delayed time to first severe exacerbation in the formoterol-treated arm. ${ }^{14} 20$ Both found a significant delay in time to first exacerbation for formoterol compared with terbutaline as rescue medication. In addition, the regular use of the short-acting terbutaline has been associated with increased bronchial hyper-reactivity and airways inflammation. ${ }^{21} 22$ Thus, some of the findings in the study by Bousquet et al might as plausibly be attributed to the pharmacological differences between as-needed bronchodilators as to the differences in combination strategies. A study by Kuna and colleagues comparing salmeterol/fluticasone plus terbutaline, budesonide/formoterol plus terbutaline and SMART was similarly confounded. ${ }^{23}$ Moreover, the patients in the salmeterol/ fluticasone arm of that study used two different delivery systems (a pressurised aerosol inhaler and a dry powder inhaler), a potential factor in the results seen.

A recent Cochrane analysis of the findings in these studies concluded that '... [SMART] can reduce the risk of asthma exacerbations needing oral corticosteroids in comparison with fixed dose maintenance inhaled corticosteroids ... [SMART] has not been demonstrated to significantly reduce exacerbations in comparison with current best practice'. ${ }^{\prime}$

\section{Level of asthma control achieved with SMART}

The goals of asthma management have evolved. The prevention of asthma death, acute hospitalisations or shortening the duration of an acute asthma episode in the emergency department are no longer regarded as sufficient treatment targets. The main focus is now on achievement of daily control and prevention of the consequences of insufficient daily control such as severe medical crises and day-to-day disability. ${ }^{14}$ Patients with asthma should be controlled so that they have few or no daytime symptoms, no night-time symptoms, rare need for acute bronchodilator relief, no physical limitations, few or no exacerbations and no absences from usual activities caused by asthma. The SMART trials have reported mean results for each of these outcomes separately rather than reporting the number of patients achieving control, defined by attaining an adequate composite score of outcome variables. A composite score is a more rigorous and discriminating evaluation of control than any single symptom parameter and has been recommended in the recent ATS/ERS statement concerning the evaluation of control in clinical practice and clinical trials. ${ }^{1324-26}$ Only recently has the attainment of asthma control been reported for SMART-treated patients. In a retrospective analysis of five trials, ${ }^{10-12} 1923$ Bateman and colleagues reported that, by the last week of the study period (6-12 months), only $17.1 \%$ of study patients were considered controlled according to Global Initiative for Asthma (GINA) criteria. ${ }^{27}$ The remaining patients were either partly controlled (38.7\%) or uncontrolled (44.2\%). Among all trial patients, the risk of exacerbation was approximately twice as high for a partly-controlled versus a controlled patient and almost six times as high for an uncontrolled versus controlled patient. 
The results from the individual asthma control outcomes in the SMART studies are summarised in table 1 and are consistent across studies. On average, patients using SMART have used quick reliever once per day, have awakened with asthma symptoms once every 7-10 days, enjoyed freedom from asthma symptoms on fewer than half of the days and experienced a severe exacerbation rate of approximately one in five patients per year (this last figure, expressed as the inverse of the annual rate of exacerbations per subject given in table 1 , will slightly overestimate the numbers of patients affected because some patients suffered more than one exacerbation during the trial). The largest effect of treatment is on exacerbations, which have been reduced by around $50 \%$ compared with comparator arms. The effect on the daily control parameters has been much smaller. The data are reported as mean values for the individual outcomes, so until recently it has not been known if a subset of very unstable patients has driven these results or in how many patients guideline-defined well-controlled disease was achieved. Consistent with this lack of day-to-day asthma control, SMART studies have not typically reported improvements in health status or quality of life relative to baseline or to comparator regimens. This is remarkable given the putative benefit of severe exacerbation reduction, an outcome of considerable importance to patients. $^{28} 29$

\section{Compliance}

It has been suggested that the SMART strategy provides greater clinical efficacy at lower doses of ICS than the traditional combination regimens, and that the corticosteroid works more effectively when inhaled at the onset of exacerbations following the onset of symptoms rather than preventively. ${ }^{30}$ These suggestions have been based on diary card records of medication use in the SMART trials but have not been validated by the use of objective measurements. Poor compliance with inhaled therapy is well documented and appears, at least in some studies, to be no better in clinical trials than in routine clinical care environments. ${ }^{31}$ Patients in trials have been reported to hide their non-compliance from investigators; in the first Lung Health Study, the phenomenon of 'dose-dumping' in patients was detected in $30 \%$ by the end of the trial and the suboptimal compliance was not detectable by diary card record or canister weighing. ${ }^{32} 33$ Electronic monitoring of dry powder devices including the Turbuhaler is available and studies using such monitoring have been reported. For example, Bosley and colleagues used this technique to assess the impact on compliance of combining a bronchodilator with an inhaled steroid. ${ }^{34}$
The treatment was taken as prescribed on only $30-40 \%$ of days, with no evidence that compliance was enhanced by the addition of terbutaline to budesonide. The authors concluded that compliance appears to be a patient-dependent rather than a drug-dependent issue.

In the SMART studies, patients have been recruited for their pattern of poorly controlled asthma despite treatment with ICS (with or without LABA). It is therefore possible that the persistence of poor day-to-day symptom control on SMART treatment, together with an apparent reduction in the need to seek medical attention for severe exacerbations, is the consequence of poorly compliant patients remaining poorly compliant with their day-today maintenance therapy but resorting to rescue combination therapy for the self-treatment of exacerbations. Further studies using objective monitoring of compliance are needed.

\section{UNANSWERED QUESTIONS}

\section{Importance of symptom perception}

SMART relies on a close association between bronchodilator use and the underlying level of asthma control. However, this relationship is known to be imperfect. Patients often choose not to use bronchodilator to relieve symptoms; asthma control indices enumerate as separate parameters the frequency of daytime or night-time symptoms and the frequency of reliever use for this reason. Some patients with asthma may be particularly insensitive to the perception of airway narrowing and the need to use bronchodilator. Rubinfield and Pain documented the phenomenon of the 'poor perceiver', finding that one in seven of their patients was unable to perceive a decrease in forced expiratory volume in $1 \mathrm{~s}$ to $<50 \%$ of predicted during methacholine challenge. ${ }^{35}$ Later investigations have identified patients who appear 'hypersensitive' to very small changes in airway calibre and may use their bronchodilator too frequently. ${ }^{36} 37$ In agreement with these findings, a poor correlation between measured airflow and as-needed bronchodilator use has been reported in patients with asthma; patterns of overuse and underuse of bronchodilator predominate with only a minority using bronchodilator appropriately. ${ }^{38}$ Misuse was linked to high anxiety scores on the Minnesota Multiphasic Personality Inventory. More studies are needed to assess the importance of these problems on the effect on symptom-driven maintenance therapy.

\section{Long-term outcomes}

A recent study has reported increased airways inflammation among patients transitioning from conventional fixed dose

Table 1 Asthma control end points, baseline and during SMART therapy

\begin{tabular}{|c|c|c|c|c|c|c|c|c|}
\hline & $\begin{array}{l}\text { Rabe } \\
\text { et } a^{10}\end{array}$ & $\begin{array}{l}\text { Scicchitano } \\
\text { et } \text { al }^{11}\end{array}$ & $\begin{array}{l}\text { 0'Byrne } \\
\text { et al }{ }^{12}\end{array}$ & $\begin{array}{l}\text { Rabe } \\
\text { et } a l^{14}\end{array}$ & $\begin{array}{l}\text { Vogelmeier } \\
\text { et } \text { al }^{15}\end{array}$ & $\begin{array}{l}\text { Kuna } \\
\text { et } a l^{21}\end{array}$ & $\begin{array}{l}\text { Bousquet } \\
\text { et } a^{19}\end{array}$ & $\begin{array}{l}\text { Weighted } \\
\text { averages }\end{array}$ \\
\hline N (SMART arm) & 354 & 947 & 925 & 1107 & 1067 & 1052 & 1151 & \\
\hline Study duration & 6 months & 12 months & 12 months & 12 months & 12 months & 6 months & 6 months & $\mathrm{N} / \mathrm{A}$ \\
\hline \multirow[t]{2}{*}{ Symptom-free days (\%) } & B: 29.6 & B: 9.8 & B: 23.1 & B: $9.2^{*}$ & NA & B: 9.3 & B: 10.7 & B: 13.2 \\
\hline & $\mathrm{T}: 55.1$ & $\mathrm{~T}: 41.7$ & $\mathrm{~T}: 54$ & $\mathrm{~T}: 40.3^{*}$ & & $\mathrm{~T}: 44.2$ & $\mathrm{~T}: 47.2$ & $\mathrm{~T}: 46.0$ \\
\hline \multirow{2}{*}{$\begin{array}{l}\text { As-needed reliever use } \\
\text { inhalations/day) }\end{array}$} & B: 1.64 & B: 1.9 & B: 2.46 & B: 1.8 & B: 2.6 & B: 2.29 & B: 2.23 & B: 2.18 \\
\hline & $\mathrm{T}: 1.04$ & $\mathrm{~T}: 0.90$ & $\mathrm{~T}: 1.01$ & $\mathrm{~T}: 1.02$ & $\mathrm{~T}: 0.59 \dagger$ & $\mathrm{T}: 1.02$ & $\mathrm{~T}: 0.95$ & $\mathrm{~T}: 0.92$ \\
\hline \multirow[t]{2}{*}{ Reliever-free days (\%) } & B: 24.3 & B: 29.3 & B: 8.2 & $\mathrm{~B}: \mathrm{N} / \mathrm{A}$ & NA & B: 8.9 & B: 10.3 & B: 14.7 \\
\hline & $\mathrm{T}: 55.3$ & $\mathrm{~T}: 59.8$ & $\mathrm{~T}: 55$ & $\mathrm{~T}: 52$ & & $\mathrm{~T}: 56$ & $\mathrm{~T}: 58.2$ & $\mathrm{~T}: 56.1$ \\
\hline \multirow[t]{2}{*}{ Nights with awakenings (\%) } & B: 13.3 & B: 22.6 & B: 21.8 & B: 31.1 & NA & B: 33.7 & B: 32.1 & B: 27.7 \\
\hline & $\mathrm{T}: 6.5$ & $\mathrm{~T}: 9.4$ & $\mathrm{~T}: 9.0$ & $\mathrm{~T}: 14.1$ & & $\mathrm{~T}: 14.1$ & $\mathrm{~T}: 12.0$ & $\mathrm{~T}: 11.5$ \\
\hline $\begin{array}{l}\text { Severe exacerbations } \\
\text { (events/patient/year) }\end{array}$ & 0.08 & 0.23 & 0.19 & 0.19 & 0.24 & 0.24 & 0.25 & 0.22 \\
\hline
\end{tabular}

*24-hour period.

†For weeks $0-4$ (during which morning and evening budesonide/formoterol doses were unchanged at two inhalations of 200/6).

$B$, baseline; T, treatment; NA, not available; N/A, not applicable; SMART, single maintenance and reliever therapy. 
Figure 2 Changes from baseline in (A) sputum cellularity and $(B)$ endobronchial biopsy cellularity following 1 year of single maintenance and reliever therapy (SMART) (budesonide/formoterol 200/6 twice daily plus as needed) or fixed dose therapy (budesonide/formoterol $800 / 12$ twice daily). Sputum and biopsy eosinophils decreased significantly with fixed dose combination therapy while with SMART there was a non-significant trend towards increased sputum eosinophils and a significant increase in biopsy eosinophils. $p$ Values are between-treatment comparisons.

maintenance therapy (when patients were receiving an average of $804 \mu \mathrm{g}$ ICS per day) to SMART therapy for 1 year prescribed as $200 / 6 \mu \mathrm{g}$ budesonide/formoterol twice daily plus as-needed doses. ${ }^{9}$ In SMART-treated patients whose daily dose of budesonide averaged $604 \mu \mathrm{g}$ per day, eosinophil numbers rose significantly in bronchial biopsies compared with baseline (see figure 2). In control patients who were randomised to a conventional maintenance regimen of constant dose 800/12 $\mu \mathrm{g}$ budesonide/ formoterol twice daily with a separate SABA reliever, sputum eosinophil counts fell and in biopsy specimens both eosinophil and mast cell numbers fell significantly.

\section{Evaluating outcomes and action planning}

The prescription of the SMART strategy does not obviate the need to monitor and adjust maintenance medications, nor does it obviate the need for action plans that would permit early selftreatment of severe exacerbations requiring prednisone or equivalent. However, it is unclear from the literature on SMART which treatment outcomes best identify when the treatment should be modified. By protocol, patients employing SMART therapy are allowed to use additional budesonide/formoterol inhaler up to eight puffs per day to relieve symptoms. Presumably, if as-needed doses are needed persistently, the treatment is deemed inadequate and the maintenance adjusted. However, the exact level and duration of as-needed use at which dosage adjustments should be made are not known. In the study by Vogelmeier et al, two different maintenance doses of budesonide/ formoterol were permitted but the protocol for dosage adjustment was not specified and appears to have been at the discretion of the investigators. ${ }^{15}$

All studies with SMART have been conducted in patients with uncontrolled asthma. The potential benefits in partially controlled asthma have not been assessed, and it seems unlikely that there would be any benefits of this regimen in controlled patients who will use as-needed bronchodilator infrequently or not at all. Indeed, this highlights the conundrum posed by the SMART strategy; it is defined by the use of as-needed medication but the frequent use of as-needed medication is inconsistent with our current definitions of control.

\section{CONCLUSIONS}

Published double-blind studies have shown that budesonide/ formoterol given in the SMART regimen is more effective than budesonide alone or than lower doses of budesonide/formoterol given in conventional fashion. It is still unclear how SMART compares with guideline-recommended amounts of combination therapy taken preventively to minimise symptoms and avoid reliever need. Guideline-recommended asthma control is rarely achieved in published SMART trials, in contrast with the reported reduction of severe exacerbations. Future studies should assess which outcomes are most useful in deciding treatment adjustments for patients inadequately controlled with the strategy. The finding of increased eosinophilic airways inflammation over 1 year of treatment is of concern and calls for further long-term studies assessing inflammation and remodelling. The recruitment of poorly controlled patients with a history of recent exacerbations despite usually effective inhaled therapy may skew the results of published SMART trials and limit their application to a more typical asthma population.

Competing interests In the past 3 years, KRC has received compensation for consulting with AstraZeneca, Boehringer-Ingelheim, CSL Behring, GlaxoSmithKline, Merck Frosst, Novartis, Nycomed, Pfizer, Roche, Schering Plough and Telacris; has undertaken research funded by AstraZeneca, Boehringer-Ingelheim, CSL Behring, Forest Labs, GlaxoSmithKline, Novartis, Parangenix, Roche and Talecris; and has participated in continuing medical education activities sponsored in whole or in part by AstraZeneca, Boehringer-Ingelheim, GlaxoSmithKline, Merck Frosst, Novartis, Nycomed, Pfizer and Talecris. NCB has lectured for or received consulting fees from GlaxoSmithKline, AstraZeneca, Altana, Merck Generics, Chiesi and TEVA and has received grant support from GlaxoSmithKline and AstraZeneca. In the past year APG has received lecture fees from AstraZeneca and GlaxoSmithKline. PWJ has received consultancy fees from GlaxoSmithKline, AstraZeneca, Almirall, Boehringer-Ingelheim and Spiration; has received lecture fees from GlaxoSmithKline; and his institution will from his time as a consultant to Novartis. Within the past 3 years, SP has received compensation for consulting with Nycomed, GlaxoSmithKline, Neolab and AstraZeneca and has given lectures sponsored by Nycomed and GlaxoSmithKline. This manuscript was conceived, researched and written by the authors without assistance from employees of the pharmaceutical industry or their agents. No professional writers participated in the preparation of the manuscript.

Provenance and peer review Not commissioned; externally peer reviewed.

\section{REFERENCES}

1. Global Initiative for Asthma (GINA). Global strategy for asthma management and prevention. http://www.ginasthma.com (accessed 9 Dec 2008).

2. Greening AP, Ind PW, Northfield $\mathrm{M}$, et al. Added salmeterol versus higher-dose corticosteroid in asthma patients with symptoms on existing inhaled corticosteroid. Lancet 1994;344:219-24.

3. Pauwels RA, Lofdahl CG, Postma DS, et al; Formoterol and Corticosteroids Establishing Therapy (FACET) International Study Group. Effect of inhaled formoterol and budesonide on exacerbations of asthma. N Engl J Med 1997;337:1405-11.

4. Bateman ED, Boushey HA, Bousquet J, et al. Can guideline-defined asthma control be achieved? The Gaining Optimal Asthma Control study. Am J Respir Crit Care Med 2004;170:836-44.

5. Selroos 0. A smarter way to manage asthma with a combination of a long-acting beta(2)-agonist and inhaled corticosteroid. Ther Clin Risk Manag 2007;3:349-59.

6. Barnes PJ. Using a combination inhaler (budesonide plus formoterol) as rescue therapy improves asthma control. BMJ 2007;335:513.

7. Humbert M, Andersson TL, Buhl R. Budesonide/formoterol for maintenance and reliever therapy in the management of moderate to severe asthma. Allergy 2008;63:1567-80.

8. Cates CJ, Lasserson TJ. Combination formoterol and budesonide as maintenance and reliever therapy versus inhaled steroid maintenance for chronic asthma in adults and children. Cochrane Database Syst Rev 2009;(2):CD007313.

9. Pavord ID, Jeffery PK, Qiu Y, et al. Airway inflammation in patients with asthma with high-fixed or low-fixed plus as-needed budesonide/formoterol. J Allergy Clin Immunol 2009;123:1083-9.

10. Rabe KF, Pizzichini E, Stallberg B, et al. Budesonide/formoterol in a single inhaler for maintenance and relief in mild-to-moderate asthma: a randomized, double-blind trial. Chest 2006;129:246-56. 
11. Scicchitano R, Aalbers R, Ukena D, et al. Efficacy and safety of budesonide/ formoterol single inhaler therapy versus a higher dose of budesonide in moderate to severe asthma. Curr Med Res Opin 2004;20:1403-18.

12. O'Byrne PM, Bisgaard H, Godard PP, et al. Budesonide/formoterol combination therapy as both maintenance and reliever medication in asthma. Am J Respir Crit Care Med 2005;171:129-36.

13. Reddel HK, Taylor DR, Bateman ED, et al. An official American Thoracic Society/ European Respiratory Society statement: asthma control and exacerbations: standardizing endpoints for clinical asthma trials and clinical practice. Am J Respir Crit Care Med 2009;180:59-99.

14. Rabe KF, Atienza T, Magyar P, et al. Effect of budesonide in combination with formoterol for reliever therapy in asthma exacerbations: a randomised controlled, double-blind study. Lancet 2006;368:744-53.

15. Vogelmeier C, D'Urzo A, Pauwels R, et al. Budesonide/formoterol maintenance and reliever therapy: an effective asthma treatment option? Eur Respir $\mathrm{J}$ 2005;26:819-28.

16. Sears MR, Boulet LP, Laviolette $M$, et al. Budesonide/formoterol maintenance and reliever therapy: impact on airway inflammation in asthma. Eur Respir $\mathrm{J}$ 2008;31:982-9

17. Anon. Clinical Study Report Synopsis [D5890L00008]: A comparison of the efficacy of Symbicort SMART (Symbicort)Turbuhaler 160/4.5 $\mu \mathrm{g} 1$ inhalation b.i.d. plus asneeded) and conventional best standard treatment for the treatment of persistent asthma in adolescents and adults. A randomized, open, parallel-group, multicentre 26 weeks study. http://www.astrazenecaclinicaltrials.com (accessed 9 Dec 2008).

18. Louis R, Joos G, Michils A, et al. A comparison of budesonide/formoterol maintenance and reliever therapy vs. conventional best practice in asthma management. Int J Clin Pract 2009;63:1479-88.

19. Bousquet J, Boulet LP. Peters MJ, et al. Budesonide/formoterol for maintenance and relief in uncontrolled asthma vs. high-dose salmeterol/fluticasone. Respir Med 2007; 101:2437-46.

20. Tattersfield AE, Lofdahl CG, Postma DS, et al. Comparison of formoterol and terbutaline for as-needed treatment of asthma: a randomised trial. Lancet 2001;357:257-61.

21. Kraan J, Koeter GH, vd Mark TW, et al. Changes in bronchial hyperreactivity induced by 4 weeks of treatment with antiasthmatic drugs in patients with allergic asthma: a comparison between budesonide and terbutaline. J Allergy Clin Immunol 1985;76:628-36.
22. Aldridge RE, Hancox RJ, Taylor DR, et al. Effects of terbutaline and budesonide on sputum cells and bronchial hyperresponsiveness in asthma. Am J Respir Crit Care Med 2000;161:1459-64.

23. Kuna $\mathbf{P}$, Peters MJ, Manjra Al, et al. Effect of budesonide/formoterol maintenance and reliever therapy on asthma exacerbations. Int J Clin Pract 2007;61:725-36.

24. Juniper EF, O'Byrne PM, Guyatt GH, et al. Development and validation of a questionnaire to measure asthma control. Eur Respir J 1999:14:902-7.

25. Nathan RA, Sorkness CA, Kosinski M, et al. Development of the asthma control test: a survey for assessing asthma control. J Allergy Clin Immunol 2004:113:59-65.

26. Bateman ED, Clark TJH, Frith $L$, et al. Rate of response of individual control measures varies and may overestimate asthma control: an analysis of the GOAL study. J Asthma 2007:44:667-73.

27. Bateman ED, Reddel HK, Eriksson G, et al. Overall asthma control: the relationship between current control and future risk. J Allergy Clin Immunol 2010;125:600-8.

28. Lloyd A, Price D, Brown R. The impact of asthma exacerbations on health-related quality of life in moderate to severe asthma patients in the UK. Prim Care Respir J 2007:16:22-7.

29. Lloyd A, Mclntosh E, Rabe KF, et al. Patient preferences for asthma therapy: a discrete choice experiment. Prim Care Respir J 2007;16:241-8.

30. Barnes PJ. Scientific rationale for using a single inhaler for asthma control. Eur Respir J 2007;29:587-95.

31. Simmons MS, Nides MA, Rand CS, et al. Unpredictability of deception in compliance with physician-prescribed bronchodilator inhaler use in a clinical trial. Chest 2000;118:290-5

32. Simmons MS, Nides MA, Rand CS, et al. Trends in compliance with bronchodilator inhaler use between follow-up visits in a clinical trial. Chest 1996;109:963-8.

33. Chapman KR. Reality-based medicine. Chest 2000;118:281-3.

34. Bosley CM, Parry DT, Cochrane GM. Patient compliance with inhaled medication: does combining beta-agonists with corticosteroids improve compliance? Eur Respir J 1994; 7:504-9.

35. Rubinfeld AR, Pain MC. Perception of asthma. Lancet 1976;1:882-4.

36. Rushford N, Tiller JW, Pain MC. Perception of natural fluctuations in peak flow in asthma: clinical severity and psychological correlates. J Asthma 1998:35:251-9.

37. Burdon JG, Juniper EF, Killian KJ, et al. The perception of breathlessness in asthma Am Rev Respir Dis 1982;126:825-8.

38. Mawhinney H, Spector SL, Heitjan D, et al. As-needed medication use in asthma usage patterns and patient characteristics. J Asthma 1993;30:61-71. 